\title{
DRIVER DISTRACTION: A NATURALISTIC OBSERVATION OF SECONDARY BEHAVIORS WITH THE USE OF DRIVER ASSISTANCE SYSTEMS
}

\author{
James R. Sayer, Mary Lynn Mefford, Kezia Shirkey, Jessica Lantz \\ Human Factors Division \\ University of Michigan Transportation Research Institute \\ Ann Arbor, Michigan, USA \\ E-mail: jimsayer@umich.edu
}

\begin{abstract}
Summary: This report describes the naturalistic observation of secondary behaviors performed by 66 drivers who took part in the Automotive Collision Avoidance System Field Operational Test (ACAS FOT). The ACAS FOT included two driver assistance systems, adaptive cruise control (ACC) and forward collision warning (FCW). Each driver participated in both baseline (no driver assistance systems for one week) and treatment conditions (both ACC and FCW available for 3 weeks). The method employed was to sub-sample video data, and code drivers' secondary behaviors using $4 \mathrm{~s}$ video clips of the driver, collected every 5 minutes. Eight-hundred and ninety video clips were reviewed and coded while participants drove manually, with conventional cruise control, ACC, and FCW. The results show that drivers who took part in the field test were no more likely to engage in secondary behaviors when driving with ACC and FCW in comparison to manual control. When the driver assistance systems became available to the participants, there was an increase in the number of conversations drivers had with passengers, probably related to the driver explaining the novel ACAS system to passengers. The results have important implications in that, at least for the duration of exposure examined, they counter the concern often raised that driver assistance systems will promote driver distraction, lapses in attention or modification in perceived risk.
\end{abstract}

\section{INTRODUCTION}

The Automotive Collision Avoidance System Field Operational Test (ACAS FOT) exposed a fleet of ten specially-equipped passenger cars to 12 months of naturalistic driving by laypersons recruited from the general driving population in Southeastern Michigan. The ACAS system that was installed on each car included both a Forward Crash Warning (FCW) system and an Adaptive Cruise Control (ACC) system. Both of these systems are supported by a forwardlooking radar, plus several other sensing, actuating, and threat-prediction features. The development and fabrication of the ACAS system was led by the General Motors Corporation in partnership with the Delphi Corporation. The project was operated under a cooperative agreement with the United States Department of Transportation. GM and Delphi conducted an extensive program of engineering tests and evaluations throughout the development process, following which the University of Michigan Transportation Research Institute (UMTRI) executed several stages of pilot testing with lay drivers that led up to the year-long FOT, a small portion of results from which are reported here (see Ervin, Sayer, LeBlanc, Bogard, Mefford, Hagan, Bareket, and Winkler, 2005). 
The goal of the FOT was to examine the suitability of the ACAS system for widespread deployment, from the dual perspectives of driving safety and driver acceptance. Since both aspects of evaluation involve complex interactions between the driver, vehicle, and roadway/traffic elements of the driving process, the requirement for naturalistic testing was considered appropriate. The FCW system is intended to warn the driver of an emerging conflict that could lead to a rear-end crash. A small set of incidents did occur during the FOT in which the FCW alert may well have helped the driver in avoiding a crash. Each of these involved an initial state of apparent distraction or misjudgment of the situation and culminated in a corrective response by the driver. However, the majority of alerts were perceived by the driver to have been unnecessary. Accordingly, driver ratings of FCW acceptance were mixed, showing a guarded degree of acceptance of the system.

The ACC system constitutes an enhancement of conventional cruise control. In addition to controlling speed at the so-called set-speed value selected by the driver, the system also automatically manages the driver-selected headway to a preceding vehicle. The ACC system performed very well in the field test and received high acceptance ratings by most participants. The consistent performance of the ACC system was seen to be effective in managing almost all conflicts, thereby enabling extended periods of engagement without the need for driver intervention.

Each vehicle in the FOT fleet was equipped with an on-board data acquisition system developed by UMTRI, yielding a large archival database that has been analyzed to determine the suitability of ACAS in terms of driving safety and driver-acceptance. The results reported here represent a random sample of 66 licensed drivers in southeastern Michigan. Drivers were in one of three age groups, 20 to 30, 40 to 50, and 60 to 70 years of age, with equal numbers of men and women. Each participant retained an instrumented vehicle for approximately four weeks (26 days). In the first week of driving, the vehicle operated simply like a production version of a 2002 Buick LeSabre (baseline condition). After the baseline period, the vehicle switched over automatically to the ACAS functionality (treatment condition), as anticipated by the driver based upon instructions that were provided by a researcher when the vehicle was picked up.

\section{METHOD}

The data acquisition system was programmed to capture a four-second-long video clip at a video sampling rate of $10 \mathrm{~Hz}$ from a camera directed towards the driver's face. These "exposure" videos were collected every five minutes, with the first exposure coming after the vehicle had been running for five minutes. Simultaneous images were also collected from a forward-scene camera at a rate of once per second. A random sample of five percent of the "exposure" clips were examined for evidence of secondary, non-driving behaviors. This five percent was stratified by week. Only clips in which the vehicle was traveling $25 \mathrm{mph}$ (the minimum speed at which FCW is active and ACC can be engaged) or faster were included in the sample. This resulted in a sample total of 890 "exposure" clips. The approach of stratifying the sample by week was utilized in order to better understand how driver behaviors may, or may not, change with exposure to the FCW and ACC systems, without the risk of obtaining a disproportionate sample from one portion of the driving experience. Because the sampling technique was random, there was no attempt made to weight the sample on the basis of individual drivers or the mileage they accrued. 
Each clip was viewed by a trained research assistant that included initial evaluations of interrater reliability. Coding was subsequently spot-checked by a researcher to ensure consistency and reliability in the coding process. A viewing tool (Figure 1) was designed to allow simultaneous viewing of digital video of the driver's face and the forward scene, along with a table that included an abbreviated coding scheme for the secondary behaviors. The randomly selected clips were loaded into a database, which was accessed from within a viewing tool. The viewing tool allowed the reviewer to replay each clip as many times as necessary, at various speeds, pause and view frame-by-frame as necessary. While viewing the face camera video, a researcher entered data into a secondary behaviors form, which in turn automatically wrote the data to a separate exposure video database. The secondary behaviors included in the coding scheme were developed in part based upon the limits of the information available. For example, there was no in-cabin video available other than the face video from which to code the frequency with which the radio, or other vehicle system, was adjusted. It is also important to note that the available data did not permit distinguishing between conversations with a passenger in the vehicle verses hands-free telephone use that did not require a headset, but this caveat is equally true for all ACAS and non-ACAS exposure clips.

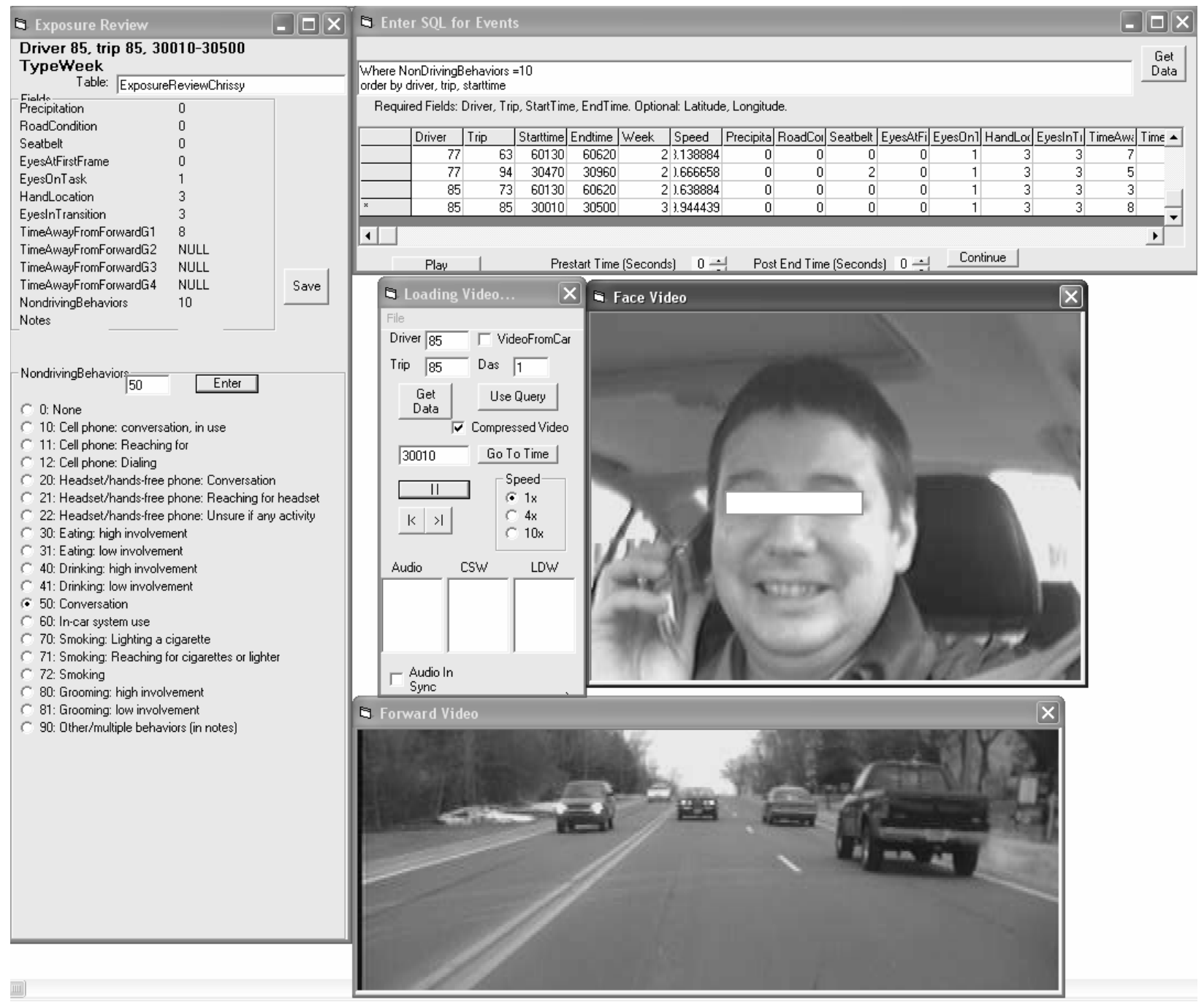

Figure 1. The viewing tool used to code videos for secondary driving behaviors. 


\section{RESULTS}

\section{Secondary Behaviors: Manual Driving with and without FCW}

For purposes of evaluating secondary, non-driving behaviors with FCW, only clips in which neither conventional nor adaptive cruise control are engaged were initially reviewed. This additional conditionality left 614 of the 890 original clips to be examined. Table 1 shows the distribution of 614 clips by age group and week.

Table 1. Counts of Exposure Clips Reviewed by Age Group and Driving Week

\begin{tabular}{|c|c|c|c|c|c|}
\hline Age Group & Week 1 & Week 2 & Week 3 & Week 4 & Total Clips \\
\hline \hline Younger & 64 & 48 & 49 & 55 & 216 \\
\hline Middle-aged & 50 & 57 & 52 & 57 & 216 \\
\hline Older & 46 & 39 & 47 & 50 & 182 \\
\hline Total Clips & 160 & 144 & 148 & 162 & 614 \\
\hline
\end{tabular}

Table 2 provides the counts of how frequently a variety of secondary behaviors were observed in the sample of exposure videos as a function of week. The bottom row of this table contains the percentages of clips in which drivers were engaged in secondary behaviors. During the first week, in manual control, drivers were engaged in secondary behaviors $18 \%$ of the time.

Table 2. Counts of exposure clips including secondary behaviors in manual control or with FCW enabled, by week

\begin{tabular}{|c|c|c|c|c|c|c|}
\hline Secondary Behavior & $\begin{array}{l}\text { Week } 1 \text { - } \\
\text { Manual }\end{array}$ & $\begin{array}{l}\text { Week } 2 \text { - } \\
\text { FCW }\end{array}$ & $\begin{array}{l}\text { Week } 3 \text { - } \\
\text { FCW }\end{array}$ & $\begin{array}{l}\text { Week } 4 \text { - } \\
\text { FCW }\end{array}$ & $\begin{array}{l}\text { FCW } \\
\text { Mean }\end{array}$ & Total Clips \\
\hline Cell phone: any activity & $11(7 \%)$ & $4(3 \%)$ & $9(6 \%)$ & $2(1 \%)$ & $5(3 \%)$ & $26(4 \%)$ \\
\hline $\begin{array}{l}\text { Hands-free cell phone: any } \\
\text { activity }\end{array}$ & $2(1 \%)$ & & $5(3 \%)$ & $1(1 \%)$ & $2(1 \%)$ & $8(1 \%)$ \\
\hline Conversation & $7(4 \%)$ & $13(9 \%)$ & $12(8 \%)$ & $14(9 \%)$ & $13(9 \%)$ & $46(7 \%)$ \\
\hline Drinking & $1(1 \%)$ & $1(1 \%)$ & $1(1 \%)$ & & $.7(1 \%)$ & $3(.5 \%)$ \\
\hline Eating & & & $1(1 \%)$ & & $.3(1 \%)$ & $1(.2 \%)$ \\
\hline Grooming & $5(3 \%)$ & $7(5 \%)$ & $3(2 \%)$ & $2(1 \%)$ & $4(3 \%)$ & $17(3 \%)$ \\
\hline Other/multiple behaviors & & $1(1 \%)$ & $1(1 \%)$ & $1(1 \%)$ & $1(1 \%)$ & $3(.5 \%)$ \\
\hline Smoking & $1(1 \%)$ & $1(1 \%)$ & $3(2 \%)$ & $3(2 \%)$ & $2.3(2 \%)$ & $8(1 \%)$ \\
\hline None & $132(83 \%)$ & $117(81 \%)$ & $112(76 \%)$ & $139(86 \%)$ & $\begin{array}{r}122.7 \\
(81 \%)\end{array}$ & $500(81 \%)$ \\
\hline Total Clips Reviewed & 160 & 144 & 148 & 162 & 151.3 & 614 \\
\hline $\begin{array}{l}\text { Clips including secondary } \\
\text { behaviors }\end{array}$ & $28(18 \%)$ & $27(19 \%)$ & $36(24 \%)$ & $23(14 \%)$ & $28.7(19 \%)$ & $114(19 \%)$ \\
\hline
\end{tabular}


During weeks 2 through 4, when the FCW system was active, drivers were observed taking part in secondary, non-driving, behaviors $19 \%$ of the time. The secondary task most frequently observed was conversation with a passenger, which was observed in approximately $9 \%$ of the exposure clips. This was followed by cell phone conversation (3\%) and grooming (3\%). It is hypothesized that the observed increase in conversation when the FCW system was enabled is quite possibly associated with the novelty of having the ACAS system and drivers' desire or excitement at the opportunity to explain how the ACAS system operated to passengers in the vehicle.

The relative frequency of observing drivers taking part in secondary behaviors has only a slight degree of variation over the four-week period, with the difference between week 1 and weeks $2-$ 4 being not statistically significant according to a Pearson $\chi^{2}$ test $\left(\chi^{2}(1, \mathrm{~N}=614)=0.16\right)$. Furthermore, other than the change in driver involvement in conversation, which is hypothesized to be associated with the novelty of having the ACAS system, the pattern and frequency of other common secondary behaviors did not appear to change. In other words, behaviors such as cell phone use and grooming appear to be equally frequent in the baseline (manual control) and treatment $(\mathrm{FCW})$ conditions. The increase in the relative frequency of conversations taking place with passengers is hypothesized to be associated with the novelty of having the FCW system, and the desire of drivers to describe the system or participation in the ACAS study to passengers. These results suggest that the presence of the FCW system did not alter the drivers' inclination to take part in secondary behaviors.

\section{Secondary Behaviors: Conventional Cruise Control verses ACC}

Since ACC is seen as a control aid that relieves the driver of the task of continuous modulation of the throttle and brake for the control of speed and headway, it has been hypothesized by some that drivers may (a) perceive a reduction in workload afforded by ACC and (b) exploit this by taking on secondary tasks they would not have performed otherwise. If it were found that drivers heavily indulged in secondary tasking when ACC is engaged, it might therefore raise a concern for a potential negative safety effect due to distraction associated with these activities.

Table 3 provides the counts of how frequently a variety of secondary behaviors were observed in the sample of exposure videos as a function of week with conventional cruise control (CCC) and ACC engaged. The 276 video clips remaining from the 5\% sample of all exposure videos included 43 in which conventional cruise control was in use (week 1) and 233 where ACC was in use (weeks $2-4$ ). The bottom row of this table contains the percentages of clips in which drivers were engaged in secondary behaviors. During the first week, while engaged in CCC, drivers were observed in secondary behaviors $7 \%$ of the time. However, this only translates to three clips in which secondary behaviors were observed. In two clips the driver was observed in a conversation with a passenger, and in one clip a driver was observed grooming.

During weeks 2 through 4, when the ACC system was engaged, drivers were observed taking part in secondary behaviors $20 \%$ of the time. The secondary task most frequently observed was a driver's conversation with a passenger, which was approximately $11 \%$ of the exposure clips, but $55 \%$ of all secondary behaviors observed with ACC engaged. This was followed by cell phone use $(3 \%)$ and grooming (3\%). Again, it is hypothesized that the observed increase in conversation when the ACC system was engaged is likely associated with the novelty of having 
the ACAS system and drivers' desire or excitement at the opportunity to explain how the system operated to passengers in the vehicle.

The rate of observing secondary tasks with ACC engaged is much higher than in the sparselyrepresented $\mathrm{CCC}$ data, but equivalent to that seen in the baseline manual driving condition and FCW treatment condition. Notwithstanding the limited sampling available for the CCC driving condition, the Pearson $\chi^{2}$ test does show that the difference seen between secondary behaviors in CCC and ACC driving is statistically significant $\left(\chi^{2}(1, N=276)=4.26\right)$. However, if one eliminates the relative increase in conversation that is hypothesized to be associated with the novelty then the change in frequency of other secondary behaviors is lessened considerably. In other words, behaviors such as cell phone use and grooming appearing with CCC engaged are only slightly, and not significantly, increased when (ACC) is engaged.

\section{Table 3. Counts of exposure clips including secondary behaviors} with CCC or ACC engaged, by week

\begin{tabular}{|c|c|c|c|c|c|c|}
\hline Secondary Behavior & $\begin{array}{l}\text { Week } 1 \text { - } \\
\text { CCC }\end{array}$ & $\begin{array}{c}\text { Week } 2 \text { - } \\
\text { ACC }\end{array}$ & $\begin{array}{c}\text { Week } 3 \text { - } \\
\text { ACC }\end{array}$ & $\begin{array}{c}\text { Week } 4 \text { - } \\
\text { ACC }\end{array}$ & $\begin{array}{l}\text { ACC } \\
\text { Mean }\end{array}$ & Total Clips \\
\hline Cell phone: any activity & & $2(3 \%)$ & $2(3 \%)$ & $3(3 \%)$ & $2.3(3 \%)$ & $7(3 \%)$ \\
\hline $\begin{array}{l}\text { Hands-free cell phone: } \\
\text { any activity }\end{array}$ & & & & & & $0(0 \%)$ \\
\hline Conversation & $2(5 \%)$ & $9(13 \%)$ & $9(12 \%)$ & $8(9 \%)$ & $9(12 \%)$ & $28(10 \%)$ \\
\hline Drinking & & & & $1(1 \%)$ & $.3(1 \%)$ & $1(.4 \%)$ \\
\hline Eating & & & & $1(1 \%)$ & $.3(1 \%)$ & $1(.4 \%)$ \\
\hline Grooming & $1(2 \%)$ & $2(3 \%)$ & $3(4 \%)$ & $1(1 \%)$ & $2(3 \%)$ & $7(3 \%)$ \\
\hline Other/multiple behaviors & & $3(1 \%)$ & $1(1 \%)$ & $1(1 \%)$ & $1.7(2 \%)$ & $5(2 \%)$ \\
\hline Smoking & & & & & & $0(0 \%)$ \\
\hline None & $40(93 \%)$ & $51(76 \%)$ & $58(79 \%)$ & $77(83 \%)$ & $62(80 \%)$ & $226(82 \%)$ \\
\hline Total Clips Reviewed & 43 & 67 & 73 & 93 & 77.7 & 276 \\
\hline $\begin{array}{l}\text { Clips including } \\
\text { secondary behaviors }\end{array}$ & $3(7 \%)$ & $16(24 \%)$ & $15(21 \%)$ & $16(17 \%)$ & $16(20 \%)$ & $50(18 \%)$ \\
\hline
\end{tabular}

\section{DISCUSSION AND CONCLUSIONS}

In summary, secondary behaviors, those behaviors that are not requisite to successfully perform the task of driving, observed when FCW was enabled and ACC was engaged occur at approximately the same rate as under manual control - but at a higher rate than under CCC. However, the conclusions with respect to comparison with conventional cruise control need to be tempered due to the very limited sample of video reviewed. Overall, the data show the following:

- Secondary behaviors were observed approximately $19 \%$ of the time while participants were driving at speeds greater than $25 \mathrm{mph}$ in the manual baseline condition, with FCW enabled, and while ACC was engaged. This suggests that drivers are no more inclined to undertake additional tasks with the driver assistance systems available than they are 
under manual control. But fewer secondary behaviors were observed when CCC was engaged (7\%), perhaps due to a limited sample or other factors related to the conditions under which drivers chose to use CCC, which have yet to be fully examined and understood.

- The higher incidence of conversation with passengers is conspicuous as the primary activity differentiating secondary activity under ACC control from that under CCC control, and FCW enabled from manual control. In fact, had an increase in conversations with passengers not been observed when FCW was enabled, an overall decline in secondary behaviors would have taken place for the FCW-enabled condition. It is hypothesized that the observed increase in conversation when either FCW or ACC was available is quite likely associated with the novelty of having the ACAS system, and drivers' desire or excitement at the opportunity to explain how the systems operated to passengers in the vehicle. This hypothesis was supported by anecdotal evidence provided by participants when they returned the research vehicles. If this increase is associated with novelty, then one might expect to see this behavior diminish as the driver assistance systems reached greater market penetration.

The significance of this work is that it represents the first naturalistic driving data set in which drivers' involvement in secondary behaviors is examined in conjunction with the use/availability of driver assistance/warning systems. These results counter the concern often raised that driver assistance/warning systems may promote driver distraction, lapses in attention or modification in perceived risk through some form of risk compensation or risk homeostatsis. This issue could be more fully understood by reviewing additional video and driver performance measures in order to determine with a greater degree of certainty what, if any, impact the use of driver assitance and warning systems might have on the instance of secondary driver behaviors. In particular, this research would have benefited from taking a larger sample of the exposure clips, particularly for the CCC condition. Driving performance measures associated with secondary behaviors can also be examined to better understand the impact of driver assistance systems on driver behavior.

\section{REFERENCES}

Ervin, R., Sayer, J., LeBlanc, D., Bogard, S., Mefford, M., Hagan, M., Bareket, Z., Winkler, C. (2005). Automotive collision avoidance system field operational test methodology and results. Unpublished technical report, The University of Michigan Transportation Research Institute, Ann Arbor, MI. 